\title{
Magnetic activity of the young solar analog V1358 Orinis
}

\author{
L. Kriskovics ${ }^{1}$, Zs. Kővári ${ }^{1,2}$, K. Vida ${ }^{1}$, K. Oláh ${ }^{1}$, T. A. Carroll ${ }^{2}$, and T. Granzer ${ }^{2}$ \\ ${ }^{1}$ Konkoly Observatory, Research Center for Astronomy and Earth Sciences, Hungarian Academy of Sciences, Budapest, Hungary \\ e-mail: kriskovics.levente@csfk.mta.hu \\ ${ }^{2}$ Leibniz Institute for Astrophysics (AIP), Potsdam, Germany
}

Received 19 December 2018 / Accepted 20 May 2019

\begin{abstract}
Context. Young, fast-rotating single stars can show dramatically different magnetic signatures and levels of magnetic activity as compared with the Sun. While losing angular momentum due to magnetic breaking and mass loss through stellar winds, the stars gradually spin down resulting in decreasing levels of activity. Studying magnetic activity on such solar analogues plays a key role in understanding the evolution of solar-like stars and allows a glimpse into the past of the Sun as well.

Aims. In order to widen our knowledge of the magnetic evolution of the Sun and solar-like stars, magnetic activity of the young solar analog V1358 Ori is investigated.

Methods. Fourier analysis of long-term photometric data is used to derive rotational period and activity cycle length, while spectral synthesis is applied to high-resolution spectroscopic data in order to derive precise astrophysical parameters. Doppler imaging is performed to recover surface-temperature maps for two subsequent intervals. Cross-correlation of the consecutive Doppler maps is used to derive surface differential rotation. The rotational modulation of the chromospheric activity indicators is also investigated.

Results. An activity cycle of $\sim 1600$ days is detected for V1358 Ori. Doppler imaging revealed a surface-temperature distribution dominated by a large polar cap with a few weaker features around the equator. This spot configuration is similar to other maps of young solar analogs from the literature, and supports recent model predictions. We detected solar-like surface differential rotation with a surface shear parameter of $\alpha=0.016 \pm 0.010$, which is in close agreement with our recently proposed empirical relation between rotation and differential rotation. The chromospheric activity indicators showed rotational modulation.
\end{abstract}

Key words. stars: activity - stars: imaging - starspots - stars: individual: V1358 Ori

\section{Introduction}

Studying magnetic activity, which is one of the indicators of the state of the underlying magnetic dynamo, is important for our understanding of the evolution of solar-like stars along the main sequence, since the magnetic dynamo strongly affects not just the stellar structure (Berdyugina 2005), but also the spin-down of the star due to magnetic breaking (e.g., Barnes 2003), angular momentum loss through stellar winds (MacGregor \& Brenner 1991), and so on.

Strong magnetic activity could also affect orbiting planetary systems through strong stellar winds or high-energy electromagnetic or particle radiation, which may ultimately erode planetary atmospheres as well (e.g., Vida et al. 2017).

Solar magnetic fields are generated by an $\alpha \Omega$-type dynamo (Parker 1955). Here, the poloidal field is wound up and amplified by the differential rotation creating the toroidal field ( $\Omega$ effect), while the $\alpha$ effect creates small-scale poloidal fields from the toroidal field via rotationally induced convective turbulence, and turbulent diffusion builds up the large-scale poloidal field by reconnection of the small-scale field.

In rapidly rotating late-type $(\mathrm{G}-\mathrm{K})$ dwarfs, the $\Omega$-effect can be suppressed, resulting in an $\alpha^{2} \Omega$ type dynamo (see Ossendrijver 2003, and references therein, or e.g., Kővári et al. 2004 for observational evidence). However, as these stars evolve, they spin down mostly due to magnetic breaking (Skumanich 1972, Barnes 2003), hence their dynamo shift from the $\alpha^{2} \Omega$ domain to the $\alpha \Omega$ (Ossendrijver 2003).

In order to properly understand the magnetic evolution of the Sun and solar-like stars along the main sequence (and its effect on their vicinity), it is imperative to study the magnetic activity of young solar-type stars.

V1358 Ori (HD 43989, HIP 30030) was originally identified as an active star and potential Doppler imaging target and classified as G0IV by Strassmeier et al. (2000). Later it was reclassified as an F9 dwarf by Montes et al. (2001), which was confirmed by McDonald et al. (2012). Vican \& Schneider (2014) estimated the effective temperature to be $T_{\text {eff }}=6100 \mathrm{~K}$. They also reported infrared excess based on WISE data and suggested the presence of a debris disk around the star. Zuckerman et al. (2011) identifies V1358 Ori as a member of the Columba association, and thus a very young $(\approx 30 \mathrm{Myr})$ solar analog.

Hackman et al. (2016) carried out a Zeeman-Doppler analysis of V1358 Ori. These latter authors reported a strong toroidal magnetic field component on the Stokes V maps, and prominent polar features on the brightness map, as well as some weaker, lower-latitude spots; however their phase-coverage was poor.

In this paper, we carry out a photometric and spectroscopic analysis of the young solar analog V1358 Ori, based on an 18-yr homogeneous photometric data set and Doppler imaging applied to high-resolution spectra covering two rotations. We also derive surface differential rotation from the consecutive Doppler maps and compare the results to similar young active solar analogs.

\section{Observations}

Strömgren $(b y)$ photometric data and Johnson-Cousins $V I_{\mathrm{C}}$ data were gathered with Wolgang and Amadeus, the $0.75 \mathrm{~m}$ automatic photoelectric telescopes operated by the Leibniz Institute 


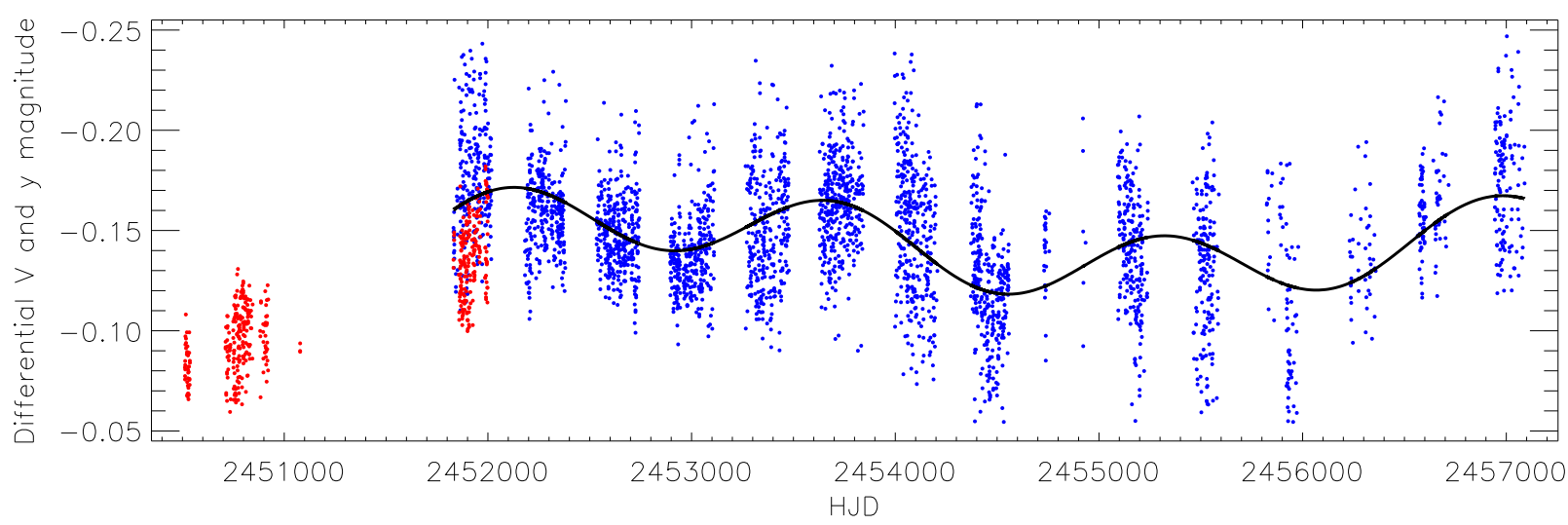

Fig. 1. Strömgren $y$ (red) and Johnson $V$ (blue) differential photometry of V1358 Ori. The overplotted curve is the combination of the two long-term cycles. See text for details.

Table 1. Spectroscopic observing log for V1358 Ori.

\begin{tabular}{cccccccc}
\hline \hline \multicolumn{3}{c}{ S1 } & & & \multicolumn{3}{c}{ S2 } \\
\cline { 1 - 4 } \cline { 6 - 8 } HJD-2456000 & $\phi$ & $S / N$ & & HJD - 2456000 & $\phi$ & $S / N$ \\
\hline 636.4313 & 0.848 & 314 & 641.4184 & 0.523 & 242 \\
636.6900 & 0.039 & 361 & 641.6738 & 0.711 & 289 \\
637.4258 & 0.581 & 332 & & 642.4252 & 0.265 & 299 \\
637.6757 & 0.765 & 321 & & 643.3967 & 0.981 & 335 \\
638.6755 & 0.502 & 342 & & 647.3944 & 0.926 & 332 \\
639.4232 & 0.053 & 312 & & 647.6529 & 0.117 & 347 \\
639.6681 & 0.233 & 317 & & & & \\
\hline
\end{tabular}

Notes. Columns S1 and S2 indicate the two subsets used for Doppler imaging; HJD $-2456000, \phi$, and $\mathrm{S} / \mathrm{N}$ are the reduced Heliocentric Julian dates, rotational phases, and $\mathrm{S} / \mathrm{N}$, respectively.

for Astrophysics Potsdam, and located at Fairborn Observatory, Arizona (Strassmeier et al. 1997) between 4 March 1997 and 8 March 2015. All differential measurements were taken with respect to the comparison star HD 44517. Between 4 March 1997 and 16 April 1997, the check star was HD 44019. After that, HD 45215 was used. For details on the data reduction, see Strassmeier et al. (1997) and Granzer et al. (2001). Photometric $V y$ data are plotted in Fig. 1. We note that there is a $0.025 \mathrm{mag}$ shift between the Stromgren $y$ data of the first two seasons and the rest of the observations, which is probably due to the different transmission characteristics of the two passbands.

Spectroscopic observations were gathered via OPTICON with the NARVAL high-resolution echelle spectropolarimeter mounted on the $2 \mathrm{~m}$ Bernard Lyot Telescope of Observatoire Midi-Pyrénées at Pic du Midi, France, between 9 and 20 December 2013 (see Table 1). A peak resolution of $R=80000$ was reached in spectroscopic object mode. The exposure time was $t_{\mathrm{exp}}=1200 \mathrm{~s}$, yielding a typical signal-to-noise ratio $(\mathrm{S} / \mathrm{N})$ of $\approx 300$. used:

To phase the observations, the following ephemeris was

$\mathrm{HJD}=2449681.5+1.3571 \times E$.

$T_{0}$ was adopted from Hackman et al. (2016). For details on the rotational period, see Sect. 3.

Spectroscopic data reduction was carried out with the standard NARVAL data-reduction pipeline. ThAr arc-lamps were used for wavelength calibration. An additional continuum fit and normalization were applied in order to avoid erroneous continuum fits during the spectral synthesis and Doppler inversion.

\section{Photometric analysis}

The period analyses were carried out on the Johnson $V$ data. Strömgren $y$ data were excluded as they only consist of 458 points and there is a long gap at the beginning of the time series (see Fig. 1).

For photometric period determination, we used MuFrAn ${ }^{1}$, a code for frequency analysis based on Fourier transformation (Kolláth 1990; Csubry \& Kolláth 2004). We accepted the peak at the cycle-per-day value of $\mathrm{c} / \mathrm{d}=0.7368$ yielding $P_{\text {rot }}=1.3571 \mathrm{~d}$ as the rotational period, which is close to the period derived by Cutispoto et al. $\left(2003, P_{\text {rot }}=1.16 \mathrm{~d}\right)$. The reasoning behind this is the following. We gradually pre-whitened the Fourier spectra with the two peaks corresponding to the two long-term changes $(\approx 1600$ and $\approx 5200$ days; see Fig. 2$)$, the suspected rotational period, and the "forest" of peaks around that value, and a signal of one day caused by the binning of the data (two or three consecutive measurements were taken in $V$ and $I$ each day). The resulting Fourier spectrum contained no signal which could reasonably be considered to be real within the precision of the photometry. Since the only period is that of $1.3571 \mathrm{~d}$ which cannot be attributed to an artificial origin or a long-term cyclic behavior, we accepted it as the rotational period. Moreover, only $P_{\text {rot }}=1.3571 \mathrm{~d}$ yields a properly phased light curve. The result is very close to the average of the seasonal periods $\left(P_{\text {rot }}=1.3618 \mathrm{~d}\right)$ and is also consistent with the measured $v \sin i$ value (see Sect. 4).

Figure 3 shows the seasonally phased $V$ light curves to demonstrate the robustness of the derived photometric period. Solid lines indicate a two-spot analytic spot model fitted to each season using SpotModel (SML; Ribárik et al. 2003). On the plot of the fourth season, both active longitudes are weak, while in the case of the seventh season, it is hard to decide which is the dominant longitude. Seasons where the low number of points made inspection impossible were omitted from the plot: two between Helicoentric Julian Dates $($ HJD) $=2454557$ and 2455092 , one between 2455239 and 2455459 , and two after 2455 588. On subplots (4) and (5), that is, intervals 2452895.0 2453111.6 and $2453265.0-2453477.6$, the active longitudes seem to shift from $\approx 0.1$ and $\approx 0.6$ to $\approx 0.3$ and $\approx 0.8$, while on subplot (10), $2455460.0-2455588.8$, they appear to be in the

\footnotetext{
1 https://konkoly.hu/staff/kollath/mufran.html
} 


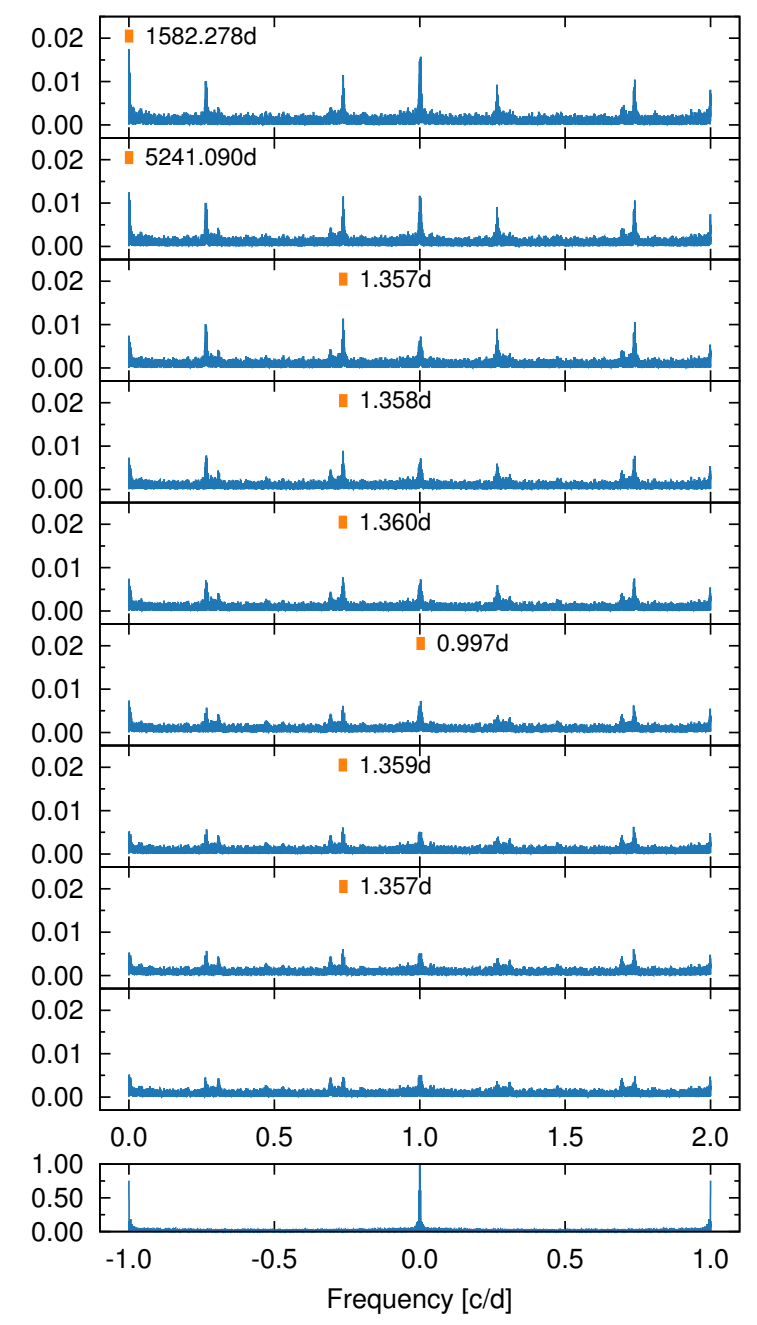

Fig. 2. Upper panels: Fourier spectra of the $V$ light curve of V1358 Ori. Orange rectangles denote the strongest peaks of the subsequent steps. Consecutive plots show pre-whitened spectra with the periods shown. Bottom panel: Fourier window function. See Sect. 3 for details.

previous positions again. This could indicate the presence of a flip-flop like phenomenon (Jetsu et al. 1994), with a time scale of $\approx 6 \mathrm{yr}$.

After visual inspection of the complete 14-yr $V$ light curve, a possible long-term cyclic behavior can be seen as well, which was confirmed by the Fourier analysis, yielding $P_{\text {cyc }} \approx 1600 \mathrm{~d}$ (see the first Fourier-spectrum plot in Fig. 2). The sum of this cycle and the other long-term change from the Fourier analysis $(\approx 5200 \mathrm{~d})$ is overplotted on the complete $V$ light curve in Fig. 1. There is a 0.025 mag systematic shift between the $V$ and $y$ data, which is probably due to the different transmission characteristics of the two bands, therefore we decided not to include the Strömgren data of the first two seasons in our long-term fit.

For further discussion on the spot configuration, suspected flip-flop, and the activity cycle, see Sect. 7.

\section{Fundamental parameters}

\subsection{Spectroscopic analysis}

Precise astrophysical parameters are fundamental for Doppler inversion, and therefore we carried out a detailed spectroscopic
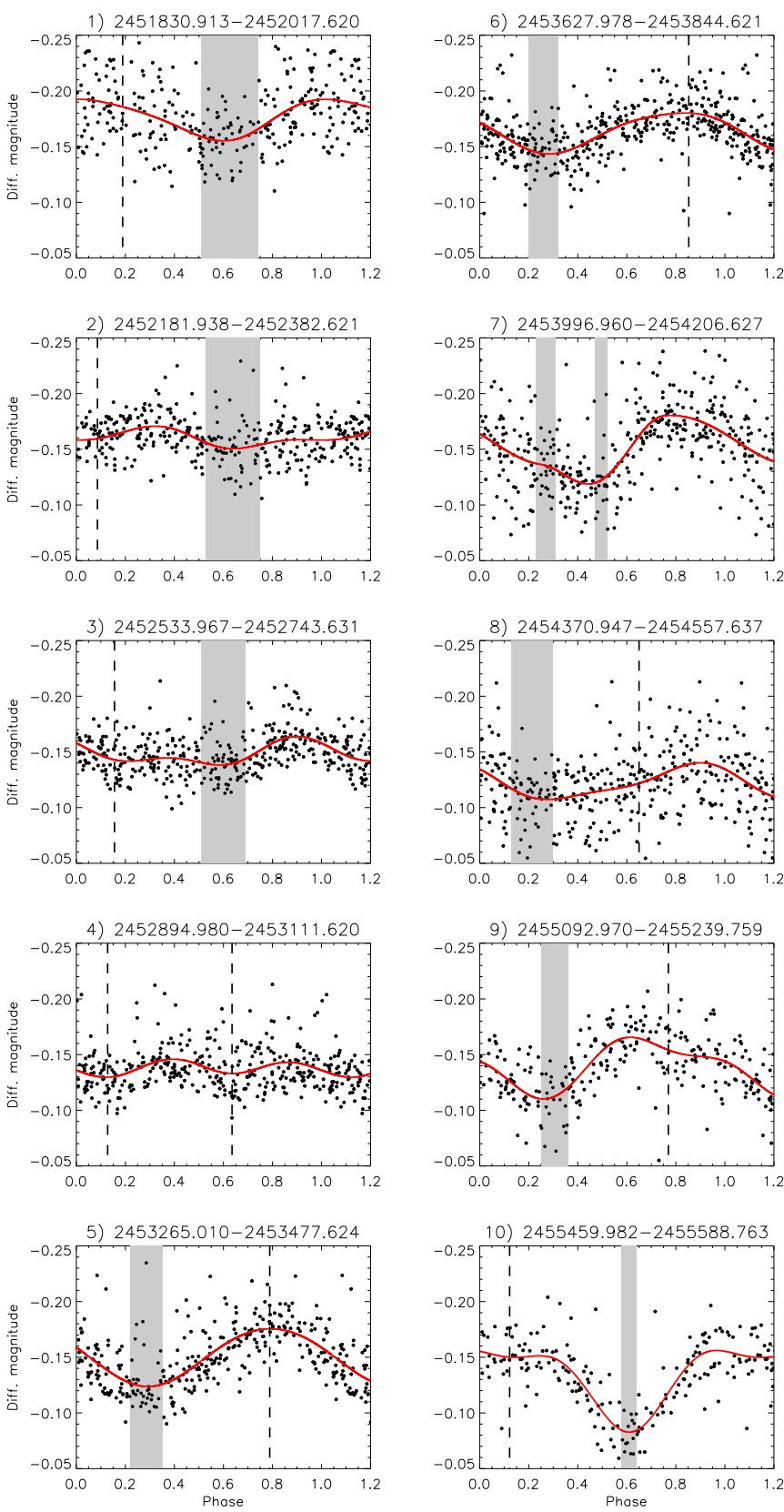

Fig. 3. Seasonal light curves of V1358 Ori phased with $P_{\text {rot }}=1.3571 \mathrm{~d}$. The time periods are denoted over the plots in JD along with the number of the seasons. Solid (red) lines show a two-spot model fitted to the light curves. Shaded zones show the longitudinal position of the dominant spot and the width of the zone denotes the error of the spot longitude. Vertical dashed lines indicate the longitude of the weaker spot. Certain seasons were omitted where a low number of data points made visual inspection meaningless.

analysis based on spectral synthesis using the code SME (Piskunov \& Valenti 2017). During the synthesis local thermodynamic equilibrium (LTE) was assumed, and MARCS models were used (Gustafsson et al. 2008). Atomic line parameters were taken from the VALD database (Kupka et al. 1999). Macroturbulence was estimated using the following equation (Valenti \& Fischer 2005):

$v_{\text {mac }}=\left(3.98-\frac{T_{\text {eff }}-5770 \mathrm{~K}}{650 \mathrm{~K}}\right) \mathrm{km} \mathrm{s}^{-1}$. 
Table 2. Fundamental astrophysical parameters of V1358 Ori.

\begin{tabular}{c|c}
\hline \hline$T_{\text {eff }}$ & $6040 \pm 25 \mathrm{~K}$ \\
$\log g$ & $4.44 \pm 0.04$ \\
{$[\mathrm{Fe} / \mathrm{H}]$} & $0.04 \pm 0.02$ \\
$v_{\text {mic }}$ & $3.0 \pm 0.5 \mathrm{~km} \mathrm{~s}^{-1}$ \\
$v_{\text {mac }}($ computed $)$ & $3.6 \mathrm{~km} \mathrm{~s}^{-1}$ \\
$v \sin i$ & $38 \pm 1 \mathrm{~km} \mathrm{~s}^{-1}$ \\
Distance & $52.0 \pm 1.3 \mathrm{pc}$ \\
$M_{\text {bol }}$ & $4.23^{\mathrm{m}+0.06}$ \\
$L / L_{\odot}$ & $1.62_{-0.07}^{+0.09}$ \\
$R / R_{\odot}$ & $1.17 \pm 0.03$ \\
Inclination & $60 \pm 10^{\circ}$ \\
$P_{\text {rot }}$ & $1.3571 \mathrm{~d}$ \\
NLTE Li abundance & $2.27 \pm 0.05$ \\
\hline
\end{tabular}

Astrophysical parameters were determined using the following methodology.

1. Determination of $v \sin i$ using initial astrophysical parameters taken from Montes et al. (2001) and assuming solar abundances.

2. Microturbulence $(\xi)$ fit with same astrophysical parameters and $v \sin i$ from step 1 using lines with $\log g f<-2.5$, since weak lines are more sensitive to the change of microturbulence.

3. Refitting $v \sin i$ and $\xi$ simultaneously to check robustness.

4. Fitting $T_{\text {eff }}$ using solar metallicity and line broadening parameters from step 3.

5. Fitting metallicity using the effective temperature value from step 4.

6. Fitting $\log g$ using stronger $\operatorname{lines}(\log g f>0)$.

7. Refitting $T_{\text {eff }}, \log g$, and metallicity simultaneously to check robustness.

8. Refitting $v \sin i$.

A lithium abundance fit was carried out using non-LTE departure coefficients (Piskunov \& Valenti 2017). The fit yielded $A(\mathrm{Li})=2.27 \pm 0.05$. An example of the fit can be seen in Fig A.1. The astrophysical parameters are summarized in Table 2.

\subsection{Distance, radius, and inclination}

The Gaia DR2 parallax of $\pi=19.22 \pm 0.05$ mas (Gaia Collaboration 2016, 2018) gives a distance of $d=52 \pm 1.3 \mathrm{pc}$. This distance using the average $V$ brightness of the star yields a bolometric magnitude of $M_{\mathrm{bol}}=4.23_{-0.05}^{+0.06 \mathrm{~m}}$ (with extinction from Schlafly \& Finkbeiner 2011 and bolometric correction from Flower 1996 taken into account). This results in a luminosity of $L / L_{\odot}=1.62_{-0.07}^{+0.09}$, which is in good agreement with the value from Gaia DR2 $\left(L / L_{\odot}=1.64 \pm 0.015\right.$, Gaia Collaboration 2018 ), and thus a radius of $R / R_{\odot}=1.17 \pm 0.03$. The radius with the photometric period and the $v \sin i=38 \pm 1 \mathrm{~km} \mathrm{~s}^{-1}$ from the spectral synthesis yields an inclination of $i=60 \pm 10^{\circ}$.

\subsection{Age}

The lithium abundance from the spectral synthesis using the empirical correlation between age and abundance from Carlos et al. (2016) would yield $t \approx 0.75 \pm 0.5 \mathrm{Gyr}$. However, it was pointed out by Balachandran (1990) and Eggenberger et al. (2010), for example, that in case of fast-rotating stars, lithium abundance is not always a suitable age indicator. Lithium depletion is also dependent on the spectral type: on an $\mathrm{M}$ dwarf, $\mathrm{Li}$ can be depleted in 10 Myrs, while on a hotter star, this rate is much lower.

According to Zuckerman et al. (2011), for example, V1358 Ori is a member of the Columba association, which has an age of $\sim 30 \mathrm{Myr}$. This is in good agreement with the computed gyrochronological age $\left(t_{\text {gyro }}=23 \pm 7\right.$ Myr using Eq. (5.3) in Barnes 2009). Therefore, it is most likely that V1358 Ori is a very young solar analog.

\section{Doppler imaging}

\subsection{The next-generation code iMap}

Our Doppler imaging code iMap (Carroll et al. 2012) carries out multi-line Doppler inversion on a list of photospheric lines between 5000 and $6750 \AA$. We included 40 virtually nonblended absorption lines with suitable line depth and temperature sensitivity, and well-defined continuum. The stellar surface is divided into $5^{\circ} \times 5^{\circ}$ segments. For each local line profile, the code uses a full radiative solver (Carroll et al. 2008). The local line profiles are then disk integrated and the individually modeled, diskintegrated lines are averaged. Atomic line data are taken from VALD (Kupka et al. 1999). Model atmospheres are taken from Castelli \& Kurucz (2004) and are interpolated for the necessary temperature, gravity, or metallicity values. Due to the high computational capacity requirements, LTE radiative transfer is used instead of spherical model atmospheres, but imperfections in the fitted line shapes are well compensated by the multi-line approach. Additional input parameters are micro- and macroturbulence, and $v \sin i$.

For the surface reconstruction, an iterative regularization method based on a Landweber algorithm was used (Carroll et al. 2012), meaning no additional constraints are imposed in the image domain. According to our tests (see Appendix A of Carroll et al. 2012 for details), the iterative regularization proved to be effective and inversions based on the same datasets always converged to the same image solution.

\subsection{Surface reconstructions}

The available 15 spectra are divided into two subsets. The corresponding time intervals are 2456636.43-2456639.66 and 2456641.42-2456647.65. The first subset consists of eight spectra and covers 2.4 rotations, while the other seven spectra of the second subset cover 4.6 rotations. The phase coverages are not completely uniform, nevertheless both subsets are suitable for Doppler imaging.

The two resulting Doppler reconstructions (henceforth S1 and S2) for V1358 Ori are plotted in Fig. 4. The average profiles are plotted along with the final profile fits (thick black and thin red lines, respectively) in Fig 5.

The overall characteristics of the two individual Doppler reconstructions are relatively similar. This is supported by the average Doppler image using all the available spectra; see Fig. 6. The resulting average map is relatively similar to the two individual images in Fig. 4, indicating only minor changes in the spot configuration.

Doppler imaging reveals a strong polar cap, as well as both cool and hot features at lower latitudes, down to the equator. Spot temperatures range from $\approx 4500 \mathrm{~K}$ to $\approx 6400 \mathrm{~K}$; this latter is $\approx 350 \mathrm{~K}$ higher than the temperature of the quiet photosphere. The contrast of the coolest features of $\approx 1500 \mathrm{~K}$ relative to the unspotted photosphere is close to the typical value for a late $\mathrm{F}$, early G dwarf (Berdyugina 2005). 

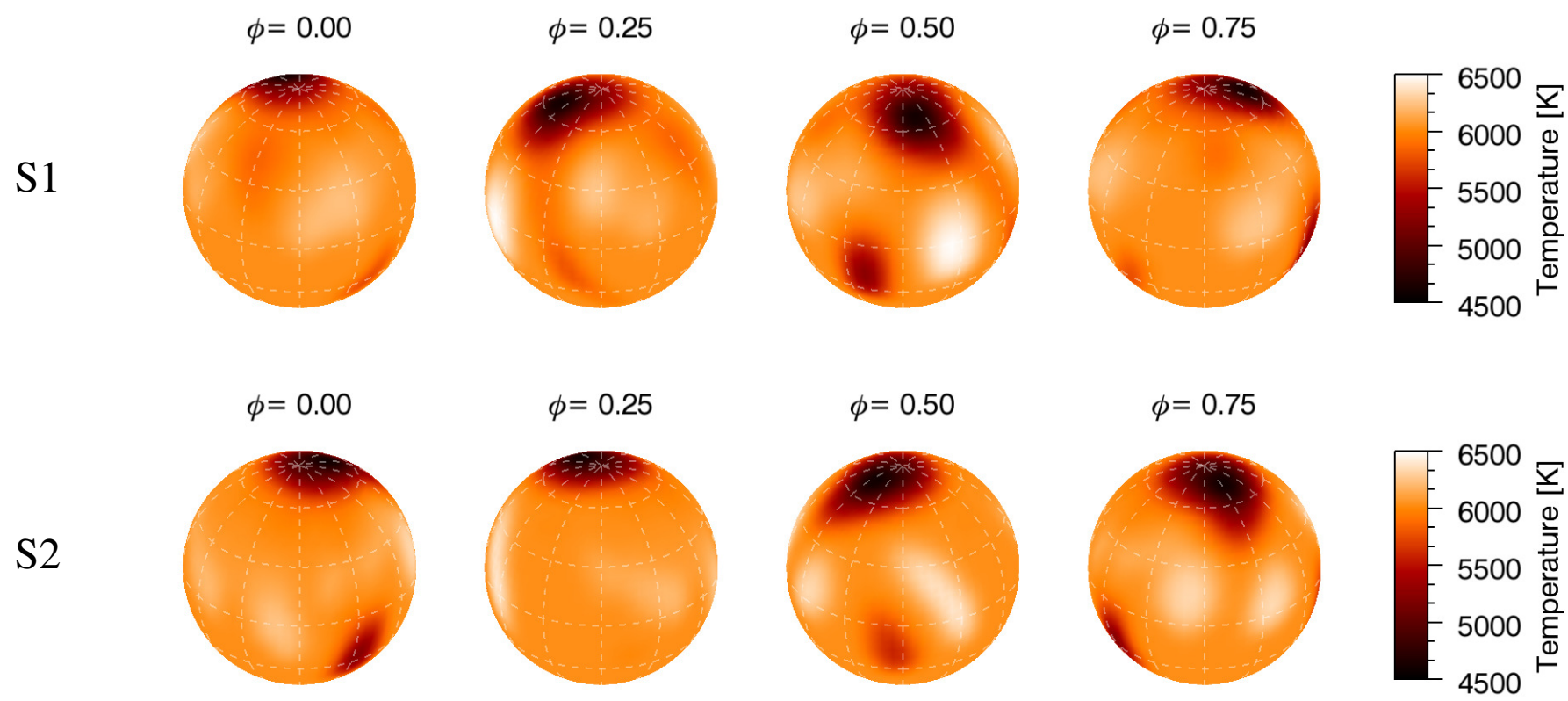

Fig. 4. Two consequent Doppler images of V1358 Ori plotted in four different rotational phases. The corresponding average HJDs for the maps are 2456638.1 and 2456653.9 for S1 and S2, respectively.
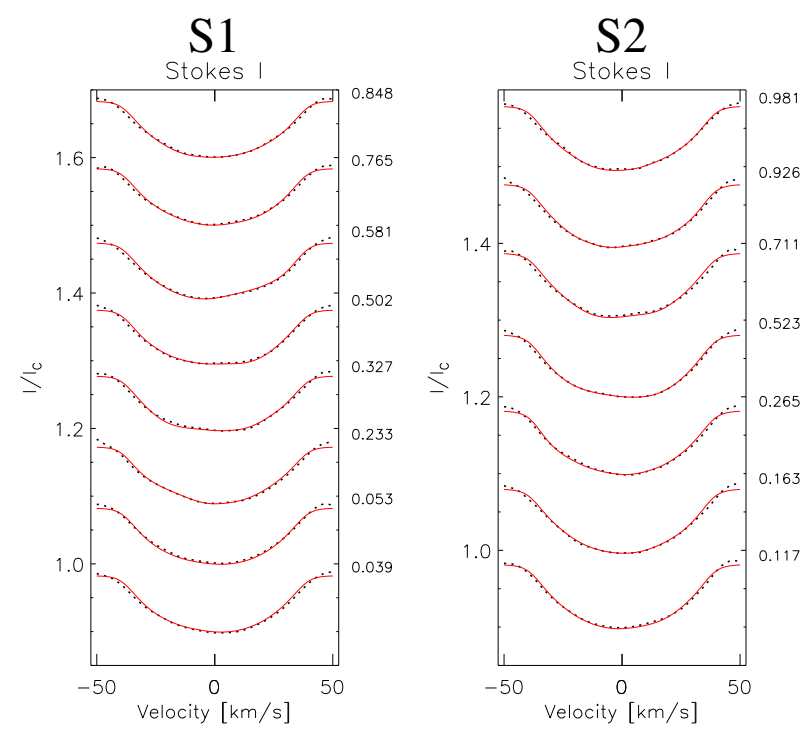

Fig. 5. Observed averaged line profiles and final fits for the two subsets. The dotted (black) lines represent the observations, while the solid (red) lines are the fits from the Doppler imaging. Rotational phases are marked on the right side of the plots.

The shape of the polar feature changes somewhat from $\mathrm{S} 1$ to $\mathrm{S} 2$ : while on $\mathrm{S} 1$, the spot features an appendage reaching $\approx 45^{\circ}$ latitude around 0.4 phase, another eccentricity is seen at $\approx 0.7$ on S2. There is a fainter cool feature around $30^{\circ}$ latitude and 0.9 phase, which almost completely disappears on S2, however the sub-equatorial spot around 0.2 phase becomes more prominent. The other cool low-latitude feature at $\approx 0.6$ phase fades somewhat, and is also displaced to $\approx 0.55$.

There are bright features present on both maps. There is a larger one at $\approx 0.4$ phase which becomes somewhat less prominent and has a different shape on the second image. There are also other much weaker equatorial bright spots on both maps, with slightly different shapes.
Hot features are often considered to be of artificial origin, mostly caused by insufficient phase coverage, as previous tests (e.g., Lindborg et al. 2014) have pointed out. However, decreasing the phase coverage usually introduces both cool and hot features on roughly the same longitude (Fig. 3 in Lindborg et al. 2014). Also, the two Doppler maps both show similar hot features at the same positions, and are based on completely independent datasets with different phase coverages. These features can also be seen on the average map derived from all of the spectra, where the phase coverage is inherently better, roughly at the same positions. The shape of the chromosperic activity indicator curves might also support the conclusion that the bright spots are real (see Sect. 6). Thus, we conclude that it is more likely that these hot spots are indeed real features.

\subsection{Differential rotation}

Longitudinal spot displacements from the first series compared to the second can be used as a tracer of surface differential rotation. Visual inspection of the two subsequent Doppler maps may indicate such rearrangements (see Sect. 5.2): the longitudinal displacement of the subequatorial cool spot around 0.4 phase or the displacement and change of shape of the bright feature at $\approx 0.6$ phase can be interpreted as such.

Surface differential rotation can be measured by longitudinally cross-correlating consecutive Doppler images (Donati \& Collier Cameron 1997), and fitting the latitudinal correlation peaks by an assumed quadratic rotational law (see e.g., Kővári et al. 2012):

$\Omega(\beta)=\Omega_{\mathrm{eq}}-\Delta \Omega \sin ^{2} \beta$,

where $\Omega(\beta)$ is the angular velocity at $\beta$ latitude, $\Omega_{\mathrm{eq}}$ is the angular velocity of the equator, and $\Delta \Omega=\Omega_{\mathrm{eq}}-\Omega_{\text {pole }}$ gives the difference between the equatorial and polar angular velocities. With these, the dimensionless surface shear parameter $\alpha$ is defined as $\alpha=\Delta \Omega / \Omega_{\mathrm{eq}}$.

We cross-correlate the available two Doppler images to build up a 2D cross-correlation function map shown in Fig. 7. 

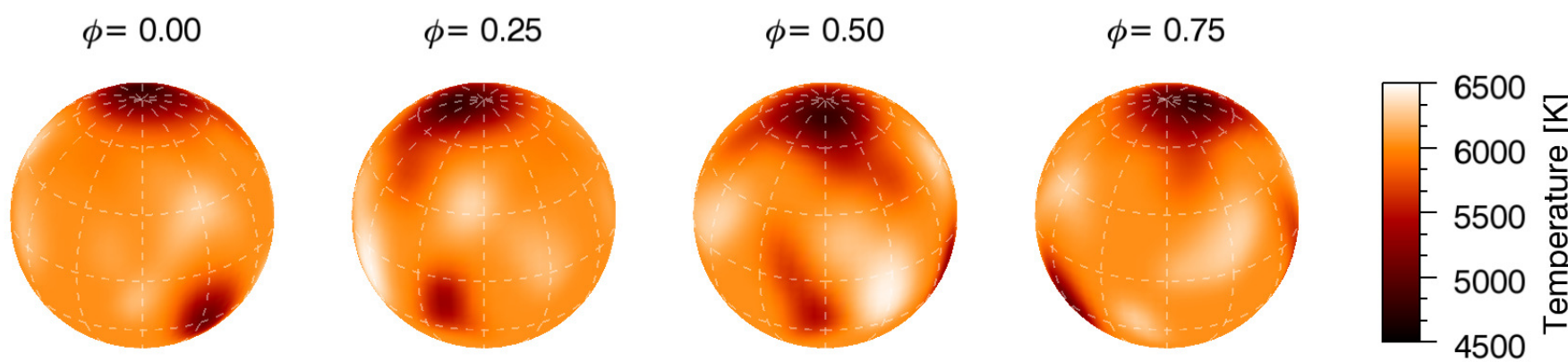

Fig. 6. Average Doppler image of V1358 Ori derived using all of the spectra plotted in four rotational phases.

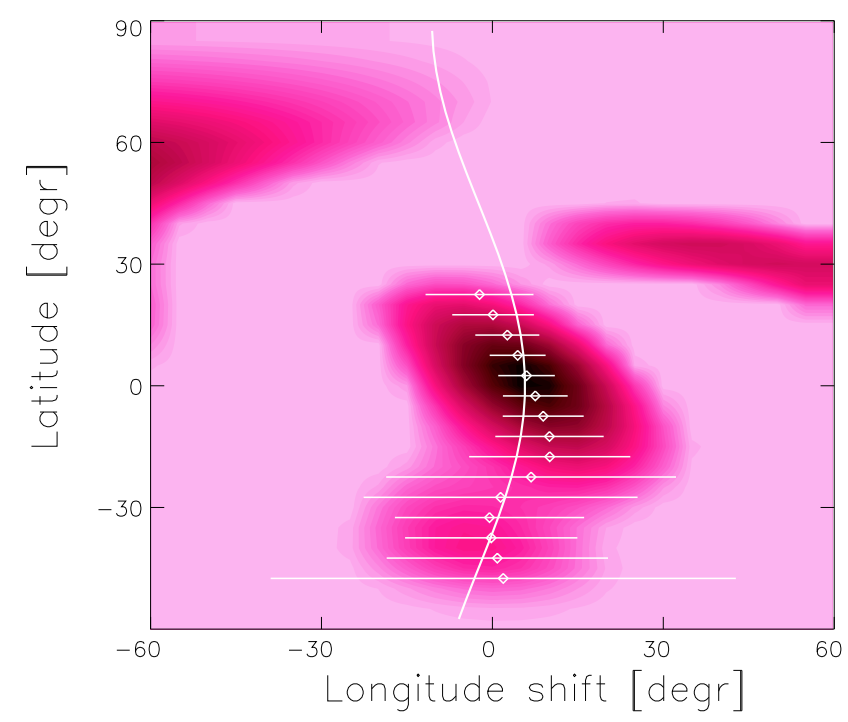

Fig. 7. Cross-correlation function map obtained by cross-correlating the two subsequent surface maps shown in Fig. 4. Darker regions correspond to stronger correlation. The best-fit rotation law to the correlation peaks (dots) suggests solar-type differential rotation with a surface shear of $\alpha=0.016$.

Despite some noise it is clear from the figure that the equator rotates most rapidly and the rotation velocity decreases with increasing latitude angle, that is, the correlation pattern indicates solar-type surface differential rotation on V1358 Ori. When fitting the pattern with a solar-type differential rotation law of the quadratic form above we get $\Omega_{\mathrm{eq}}=266.8 \pm 0.3 \%$ day and $\Delta \Omega=4.3 \pm 1.0 \%$ day resulting in a surface shear parameter of $\alpha=0.016 \pm 0.004$. Errors are estimated from the FWHMs and amplitudes of the Gaussian fits to the latitudinal bins. However, having only two consecutive Doppler images could introduce false correlation, making the cross-correlation technique less powerful (Kővári et al. 2017). Moreover, due to projection effects, the actual impact of the strongest polar features is restricted and the low-latitude features are less contrasted. As a result, the true errors of the derived surface shear may be somewhat larger, therefore we assume error bar of \pm 0.01 for $\alpha$ instead of the formal value of \pm 0.004 from the fit. For further discussion on the differential rotation, see Sect. 7 .

\section{Chromospheric activity}

We measured the Ca II $R_{\mathrm{HK}}, \mathrm{H} \alpha$, and Ca II infrared triplet (IRT) chromospheric activity indices for all of the spectra individually to see if there is any rotational modulation present for the two covered rotations.
For the $R_{\mathrm{HK}}$, we first calculated the noncalibrated $S$-index as described in Vaughan et al. (1978). The instrumental values were then transformed into the original Mt. Wilson scale with the calibration coefficients for NARVAL derived by Marsden et al. (2014). To avoid color-dependence, $S$-index values were transformed to $R_{\mathrm{HK}}$ (Middelkoop 1982; Rutten 1984). To subtract the photospheric adjunct, we applied the correction formula of Noyes (1984). For the $\mathrm{H} \alpha$, we used the indicator defined by Kürster et al. (2003). The IRT index was calculated using the formula of Marsden et al. (2014).

The apparent average surface-temperatures were also computed for the two Doppler images in the same phases for comparison. The $R_{\mathrm{HK}}, \mathrm{H} \alpha$, and the IRT indices are plotted in Fig. 8 along with average temperatures. The values themselves are summarized in Table 3 . The errors were estimated using error propagation and are on the order of $0.004,0.003$, and 0.001 for $R_{\mathrm{HK}}, \mathrm{H} \alpha$, and IRT, respectively.

All of the indices clearly show some change with the rotational phases which could be interpreted as rotational modulation. Apart from a few outlying points, the $R_{\mathrm{HK}}, \mathrm{H} \alpha$, and IRT indices show roughly the same behavior. The overall shape of the curves is similar, but there is no clear correlation between the position of the maximal chromospheric activity and the highest spot coverage (i.e., the lowest average surface-temperature). One might argue that the largest difference between the chromospheric activity and the average surface-temperature is around 0.2-0.4 phase, where, on the Doppler images, the strongest hot spot becomes gradually visible, which might indicate that the hot structure has a chromospheric counterpart (and further strengthen the hypothesis that these features are indeed not artifacts of the Doppler imaging process).

\section{Discussion}

Both visual inspection and the Fourier analysis suggest a possible activity cycle of $\approx 1600 \mathrm{~d}$, that is, $\approx 4.5 \mathrm{yr}$. The additional Strömgren $y$ photometry (see Fig. 1) also seems to confirm this cycle, but these data were not included in the Fourier analysis, as the Strömgren $y$ filter is much narrower than the Johnson $V$ (23 vs. $88 \mathrm{~nm}$ ) and there is also a large gap in those observations. The rotational period and the length of the activity cycle are two important observables of magnetically active stars. Their ratio is related to the dynamo number which is an indicator of magnetic activity. The derived rotational period and the long-term cycles (including the suspected one of $\approx 5200$ days) both show a close fit to the rotational period-activity cycle length relation derived by Oláh et al. (2016) (see Fig. 5 and details therein).

In subplot (3), HJD = 2452533 - 2452473 , (4), 2452894 2453 111, and (8), 2454370-2 454557 of Fig. 3, the amplitudes of the light curves are significantly lower than in the other 
Table 3. Chromospheric activity indices of V1358 Ori in the observed rotational phases.

\begin{tabular}{|c|c|c|c|c|c|c|c|}
\hline \multicolumn{4}{|c|}{$\mathrm{S} 1$} & \multicolumn{4}{|c|}{$\mathrm{S} 2$} \\
\hline$\phi$ & $\log R_{\mathrm{HK}}$ & $\log I_{\mathrm{H} \alpha}$ & $\log I_{\mathrm{IRT}}$ & $\phi$ & $\log \mathrm{R}_{\mathrm{HK}}$ & $\log I_{\mathrm{H} \alpha}$ & $\log I_{\mathrm{IRT}}$ \\
\hline 0.039 & -4.294 & -1.467 & -0.268 & 0.117 & -4.320 & -1.459 & -0.270 \\
\hline 0.053 & -4.287 & -1.470 & -0.273 & 0.163 & -4.315 & -1.472 & -0.263 \\
\hline 0.233 & -4.271 & -1.458 & -0.264 & 0.265 & -4.280 & -1.449 & -0.262 \\
\hline 0.327 & -4.272 & -1.451 & -0.261 & 0.523 & -4.285 & -1.452 & -0.262 \\
\hline 0.502 & -4.277 & -1.455 & -0.269 & 0.711 & -4.275 & -1.444 & -0.262 \\
\hline 0.581 & -4.284 & -1.453 & -0.261 & 0.926 & -4.298 & -1.453 & -0.267 \\
\hline 0.765 & -4.307 & -1.461 & -0.268 & 0.981 & -4.337 & -1.462 & -0.264 \\
\hline 0.848 & -4.318 & -1.478 & -0.272 & & & & \\
\hline
\end{tabular}

Notes. See Sect. 6 for more details.
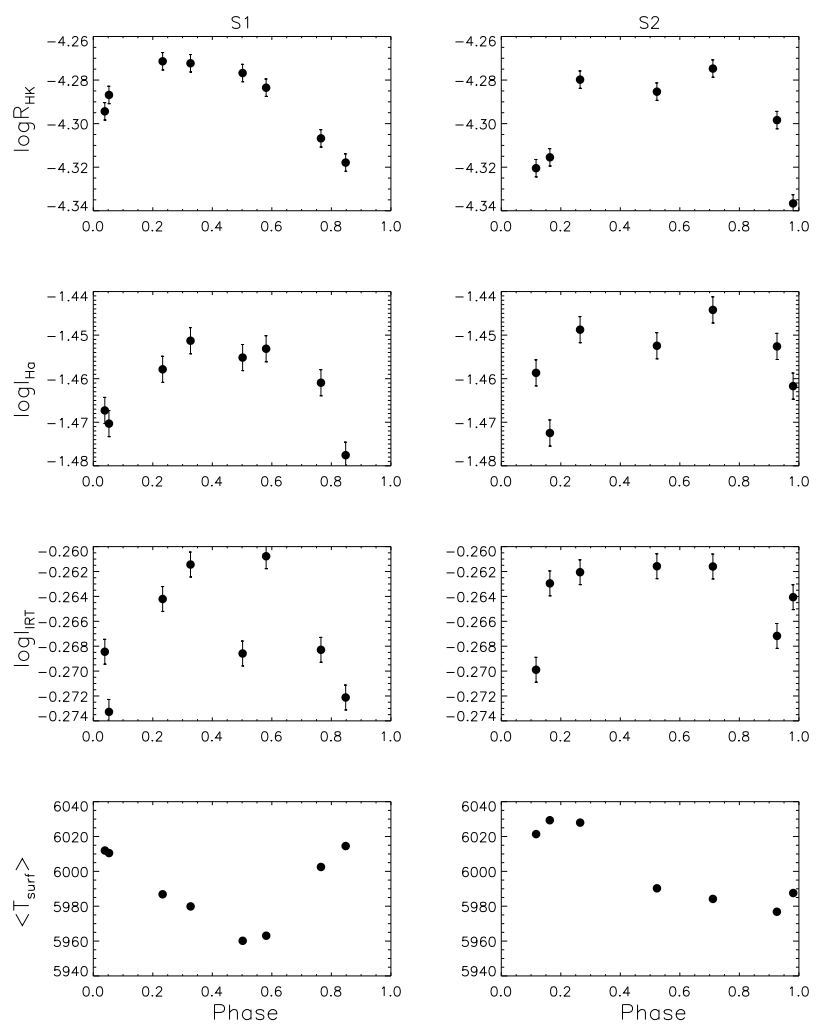

Fig. 8. Calcium $R_{\mathrm{HK}}$ (top panels), $\mathrm{H} \alpha$ (second row), and Ca II IRT (third row) curves for the two rotations compared to the average projected surface-temperatures for the same rotational phases from Doppler imaging (bottom panels).

seasons. This may be a prelude to the actual flip-flop phenomenon: the stronger active region gradually weakens, while the weaker one becomes stronger.

The suspected flip-flop period of $\sim 6 \mathrm{yr}$ would fit the flip-flop periods found on single dwarfs by Elstner \& Korhonen (2005). These latter authors pointed out that the presence of the flip-flop mechanism shows little to no dependence on the thickness of the convective layer (and hence the spectral type), however they suspected a stronger dependence on the strength of the surface differential rotation. These authors also found that the reported flip-flop periods of several years $(\approx 4-9)$ are found in the range of $|\alpha|=\left|\Delta \Omega / \Omega_{\text {eq }}\right| \approx 0.015-0.15$ (see Elstner \& Korhonen 2005 and references therein). Our values $\left(\alpha=0.016 \pm 0.01\right.$ and $\left.P_{\mathrm{ff}} \approx 6 \mathrm{yr}\right)$ fit this suspected relation well.
We note, however, that between the last two plotted seasons, a season was omitted due to the low number of points, therefore the above period is an upper estimate.

The surface of V1358 Ori is dominated by a large polar structure of high contrast accompanied by a low number of lowlatitude, weaker features. While polar features are not uncommon on active stars in general, this configuration seems to be a recurring phenomenon on young, single solar analogs. Järvinen et al. (2008) reported a very similar and rather stable surface structure on the young G2V star V889 Her using Doppler imaging, and later Kővári et al. (2011) derived a similar surface structure. Marsden et al. (2005) also detected a dominant polar cap without any significant low-latitude features on V557 Car, a roughly 40 Myr-old G2 solar analog. EK Dra, another young G2V star, was also reported to exhibit these features (although here with some stronger low-latitude spots as well) by Järvinen et al. (2007). Hackman et al. (2016) also derived strong polar structures and weaker equatorial features (and magnetic field configurations consistent with this picture) for V1358 Ori and another two young solar-like stars, AH Lep and HD 29615 (G3V and $\mathrm{G} 2 \mathrm{~V}$, respectively), using Zeeman-Doppler imaging. It should be noted however that on the maps of Hackman et al. (2016), there were no bright features at all around the equator, which may indicate that the magnetic configuration has undergone structural changes. Recently, Işık et al. (2018) used numerical simulations of magnetic flux transport and emergence to model the difference in latitudinal spot distribution between the Sun and other stars. They found that as the rotation rate increases, magnetic flux emerges at higher latitudes, and a quiet region opens around the equator. They also pointed out that at eight times the rotational rate of the Sun $\left(P_{\text {rot }} \approx 3 \mathrm{~d}\right)$ polar regions can form, while the width of the inactive region around the equator increases. Our findings are also consistent with this model.

Finally, we emphasize that our result of differential rotation is in agreement with the observation that active young solar-type stars exhibit weak solar-type differential rotation; for example, LQ Hya $\alpha=0.0056$ with $P_{\text {rot }}=1.597$ d, Kővári et al. 2004 , or AB Dor $\alpha \approx 0.006$ with $P_{\text {rot }}=0.5148 \mathrm{~d}$, Jeffers et al. 2007. Moreover, Kővári et al. (2011) derived $\alpha=0.009$ for V889 Her, with a rotational period of 1.337 days and Marsden et al. (2005) reported a surface shear of 0.012 on V557 Car $\left(P_{\text {rot }}=0.557 \mathrm{~d}\right)$. Our result is also in good agreement with the empirical relation between the rotational period and the surface shear parameter of

$|\alpha| \approx 0.005 P_{\text {rot }}($ days $)$ 
for single stars suggested by Kővári et al. (2017), see Fig. A.2.

The rotational modulation of the chromospheric activity indicators suggests that the positions of the chromospheric structures (plages?) more or less coincide with the positions of the photospheric nests. The most apparent difference in the shape of the curves is in the region of $0.2-0.4$ phase, which coincides with the phase where the most prominent hot feature on the Doppler images of both rotations becomes visible due to the rotation of the star. This may mean that the photospheric hot features extend to the chromosphere as well, and contribute to the overall chromospheric emission.

\section{Summary}

We carried out detailed photometric and spectroscopic analysis of the young solar analog V1358 Ori.

- Based on a 14-yr photometric dataset we derive a rotational period of $P_{\text {rot }}=1.3571 \mathrm{~d}$ for V1358 Ori.

- An activity cycle with a period of roughly 1600 days is detected, which is consistent with the findings of Oláh et al. (2016). A flip-flop time-scale of 6 yr may also be present.

- Using a spectral synthesis technique we determine precise astrophysical parameters for V1358 Ori.

- We perform Doppler imaging to map the surfacetemperature distribution for two subsequent epochs separated by two weeks. The surface structure is dominated by a large polar cap accompanied with weaker features at low latitudes, consistent with previous observations of young solar analogs and recent dynamo models as well. Hot features are also present on both maps.

- Surface differential rotational is derived by cross-correlating the two subsequent Doppler images. The resulting surface shear parameter $\alpha=0.016 \pm 0.01$ is in agreement with the rotational period-surface shear empirical relationship proposed recently by Kővári et al. (2017).

- Chromospheric activity indicators are calculated and compared to the average apparent surface-temperatures. Rotational modulation is present on the activity indicator curves in both rotations. The shapes of the curves are similar. The most prominent difference between the activity indicator curves and the average surface-temperatures may indicate that the hot spots contribute to the chromospheric emission.

Acknowledgements. The authors acknowledge the Hungarian National Research, Development and Innovation Office grant OTKA K-113117, and supports through the Lendület-2012 Program (LP2012-31) of the Hungarian Academy of Sciences, and the ESA PECS Contract No. 4000110889/14/NL/NDe. K.V. is supported by the Bolyai János Research Scholarship of the Hungarian Academy of Sciences. The authors thank A. Moór for the useful conversations on stellar ages. Finally, the authors would like to thank the anonymous referee for their valuable insight.

\section{References}

Balachandran, S. 1990, ApJ, 354, 310

Barnes, S. A. 2003, ApJ, 586, 464
Barnes, S. A. 2009, in The Ages of Stars, eds. E. E. Mamajek, D. R. Soderblom, \& R. F. G. Wyse, IAU Symp., 258, 345

Berdyugina, S. V. 2005, Liv. Rev. Sol. Phys., 2, 8

Carlos, M., Nissen, P. E., \& Meléndez, J. 2016, A\&A, 587, A100

Carroll, T. A., Kopf, M., \& Strassmeier, K. G. 2008, A\&A, 488, 781

Carroll, T. A., Strassmeier, K. G., Rice, J. B., \& Künstler, A. 2012, A\&A, 548, A95

Castelli, F., \& Kurucz, R. L. 2004, IAU Symp. 210, poster A20

Csubry, Z., \& Kolláth, Z. 2004, in SOHO 14 Helio- and Asteroseismology:

Towards a Golden Future, ed. D. Danesy, ESA S. P., 559, 396

Cutispoto, G., Messina, S., \& Rodonò, M. 2003, A\&A, 400, 659

Donati, J.-F., \& Collier Cameron, A. 1997, MNRAS, 291, 1

Eggenberger, P., Maeder, A., \& Meynet, G. 2010, A\&A, 519, L2

Elstner, D., \& Korhonen, H. 2005, Astron. Nachr., 326, 278

Flower, P. J. 1996, ApJ, 469, 355

Gaia Collaboration, Prusti, T., et al. 2016, A\&A, 595, A1

Gaia Collaboration (Brown, A. G. A., et al.) 2018, A\&A, 616, A1

Granzer, T., Weber, M., \& Strassmeier, K. G. 2001, Astron. Nachr., 322, 295

Gustafsson, B., Edvardsson, B., Eriksson, K., et al. 2008, A\&A, 486, 951

Hackman, T., Lehtinen, J., Rosén, L., Kochukhov, O., \& Käpylä, M. J. 2016, A\&A, 587, A28

Işık, E., Solanki, S. K., Krivova, N. A., \& Shapiro, A. I. 2018, A\&A, 620, A177

Järvinen, S. P., Berdyugina, S. V., Korhonen, H., Ilyin, I., \& Tuominen, I. 2007, A\&A, 472, 887

Järvinen, S. P., Korhonen, H., Berdyugina, S. V., et al. 2008, A\&A, 488, 1047

Jeffers, S. V., Donati, J.-F., \& Collier Cameron, A. 2007, MNRAS, 375, 567

Jetsu, L., Tuominen, I., Grankin, K. N., Mel'Nikov, S. Y., \& Schevchenko, V. S. 1994, A\&A, 282, L9

Kővári, Zs., Strassmeier, K. G., Granzer, T., et al. 2004, A\&A, 417, 1047

Kővári, Zs., Frasca, A., Biazzo, K., et al. 2011, in Physics of Sun and Star Spots, eds. D. Prasad Choudhary, \& K. G. Strassmeier, IAU Symp., 273, 121

Kővári, Zs., Korhonen, H., Kriskovics, L., et al. 2012, A\&A, 539, A50

Kővári, Zs., Oláh, K., Kriskovics, L., et al. 2017, Astron. Nachr., 338, 903

Kolláth, Z. 1990, Konkoly Observatory Occasional Technical Notes, 1, 1

Kupka, F., Piskunov, N., Ryabchikova, T. A., Stempels, H. C., \& Weiss, W. W. 1999, A\&AS, 138, 119

Kürster, M., Endl, M., Rouesnel, F., et al. 2003, A\&A, 403, 1077

Lindborg, M., Hackman, T., Mantere, M. J., et al. 2014, A\&A, 562, A139

MacGregor, K. B., \& Brenner, M. 1991, ApJ, 376, 204

Marsden, S. C., Waite, I. A., Carter, B. D., \& Donati, J.-F. 2005, MNRAS, 359, 711

Marsden, S. C., Petit, P., Jeffers, S. V., et al. 2014, MNRAS, 444, 3517

McDonald, I., Zijlstra, A. A., \& Boyer, M. L. 2012, MNRAS, 427, 343

Middelkoop, F. 1982, A\&A, 107, 31

Montes, D., López-Santiago, J., Gálvez, M. C., et al. 2001, MNRAS, 328, 45

Noyes, R. W. 1984, Adv. Space Res., 4, 151

Oláh, K., Kővári, Zs., Petrovay, K., et al. 2016, A\&A, 590, A133

Ossendrijver, M. 2003, A\&ARv, 11, 287

Parker, E. N. 1955, ApJ, 122, 293

Piskunov, N., \& Valenti, J. A. 2017, A\&A, 597, A16

Ribárik, G., Oláh, K., \& Strassmeier, K. G. 2003, Astron. Nachr., 324, 202

Rutten, R. G. M. 1984, A\&A, 130, 353

Schlafly, E. F., \& Finkbeiner, D. P. 2011, ApJ, 737, 103

Skumanich, A. 1972, ApJ, 171, 565

Strassmeier, K. G., Boyd, L. J., Epand, D. H., \& Granzer, T. 1997, PASP, 109, 697

Strassmeier, K., Washuettl, A., Granzer, T., Scheck, M., \& Weber, M. 2000, A\&AS, 142, 275

Valenti, J. A., \& Fischer, D. A. 2005, ApJS, 159, 141

Vaughan, A. H., Preston, G. W., \& Wilson, O. C. 1978, PASP, 90, 267

Vican, L., \& Schneider, A. 2014, ApJ, 780, 154

Vida, K., Kövári, Zs., Pál, A., Oláh, K., \& Kriskovics, L. 2017, ApJ, 841, 124

Zuckerman, B., Rhee, J. H., Song, I., \& Bessell, M. S. 2011, ApJ, 732, 61 


\section{Appendix A: Additional figures}

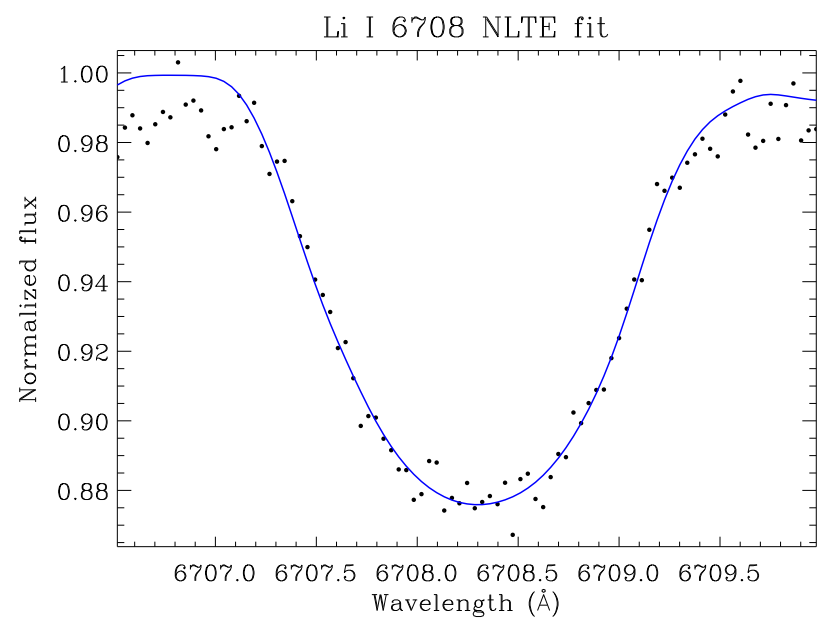

Fig. A.1. Example of an NLTE Li I 6708 fit. The corresponding lithium abundance is $A_{\mathrm{NLTE}}(\mathrm{Li})=2.17 \pm 0.03$.

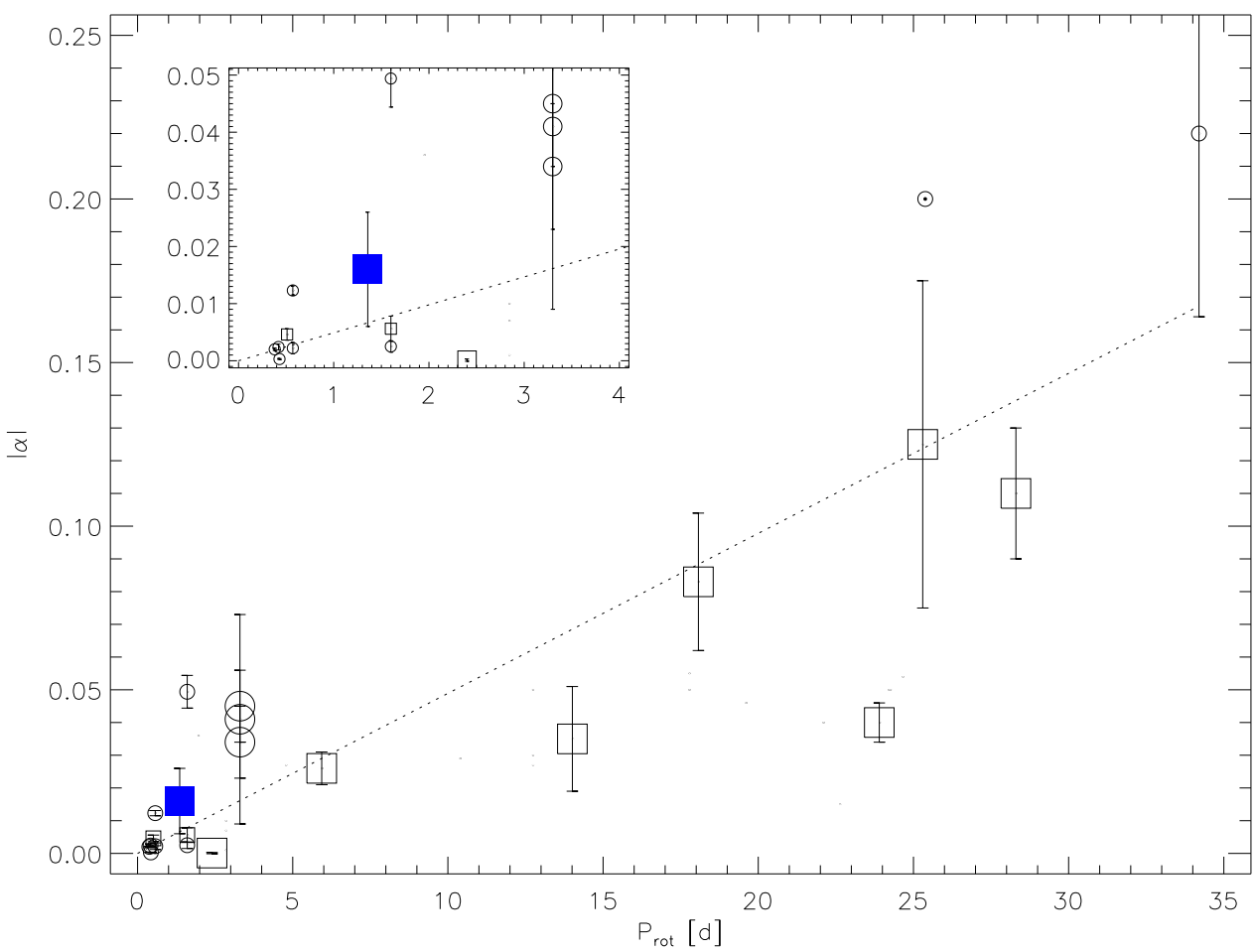

Fig. A.2. Absolute value of the dimensionless surface shear parameter of single stars plotted against their rotational period in days (see Kővári et al. 2017 and references therein). Circles denote results from the sheared image method, while squares indicate values obtained with the crosscorrelation technique. The blue filled square indicates V1358 Ori. The dotted line represents a linear fit with a steepness of $\approx 0.005$. See Sect. 7 for further details. 